B. Runge

Nagoya Math. J.

Vol. 138 (1995), 179-197

\title{
ON SIEGEL MODULAR FORMS \\ PART II
}

\section{BERNHARD RUNGE}

\section{Introduction}

In this paper we compute dimension formulas for rings of Siegel modular forms of genus $g=3$. Let denote $\Gamma_{g}(2)$ the main congruence subgroup of level two, $\Gamma_{g, 0}(2)$ the Hecke subgroup of level two and $\Gamma_{g}$ the full modular group. We give the dimension formulas for genus $g=3$ for the above mentioned groups $\Gamma$ and determine the graded ring $A\left(\Gamma_{3}(2)\right)$ of modular forms with respect to $\Gamma_{3}(2)$.

The dimension formula in the case $\Gamma=\Gamma_{3}$ was first given by Tsuyumine in [T1]. Tsuyumine, following a method of Igusa, used the sequence

$$
0 \rightarrow \chi_{18} A\left(\Gamma_{3}\right) \rightarrow A\left(\Gamma_{3}\right) \rightarrow S(2,8)
$$

where $\chi_{18}$ is a cusp form of weight 18 defining the closure of the hyperelliptic locus and $S(2,8)$ is the graded ring of invariants of binary 8 -forms. Tsuyumine uses the structure of $S(2,8)$, given by Shioda [Sh], and restriction of a bigger ring $A^{\prime}\left(\Gamma_{3}\right)$ with respect to a second divisor.

For our generalization of Tsuyumine's result we use a more direct approach. In $[R]$ we computed the ring of modular forms for $\Gamma_{3}(2,4)$ (the Igusa subgroup of level two). Principally this allows to compute all rings of modular forms for subgroups $\Gamma$ with $\Gamma_{3}(2,4) \subset \Gamma \subset \Gamma_{3}$. However, this involves subtle computations of rings of invariants with respect to the finite group $\Gamma / \Gamma_{3}(2,4)$. It turns out that the computation is simplified by constructing a certain central extension $H_{g}$ of $\Gamma_{g} / \Gamma_{g}(2,4)$. This group seems to be of independent interest because of its importance in coding theory. The main ingredient is a decomposition of Bruhat type for the group $H_{g}$. This decomposition is closely connected with the theory of partial Fourier transformation.

Finally, in the last chapter we give a characterization of decomposable points in the Satake compactification, which gives another method for computing $A\left(\Gamma_{3}(2)\right)$.

\footnotetext{
Received September 28. 1993.
} 


\section{A decomposition of Bruhat type}

We give two descriptions of the group $H_{g}$. For the first description consider

$$
V=\left\{f: \mathbf{F}_{2}^{g} \rightarrow \mathbf{C}\right\}=L^{2}\left(\mathbf{F}_{2}^{g}\right)
$$

together with the standard scalar product $\langle\rangle:, \mathbf{F}_{2}^{g} \times \mathbf{F}_{2}^{g} \rightarrow \mathbf{F}_{2}$. Let $f_{a}$ for $a \in \mathbf{F}_{2}^{g}$ be the standard basis of $V$. We regard Fourier transformation on $V$, i.e.

$$
\hat{f}(a)=2^{-g} \sum_{b \in \mathbf{F}_{2}^{g}} f(b)(-1)^{\langle a, b\rangle}
$$

and the convolution

$$
f * g(a)=2^{-g} \sum_{b \in \mathbf{F}_{2}^{g}} f(b) g(a-b) .
$$

We have the obvious properties $\overline{\hat{f}}(a)=2^{-g} f(-a)$ and $\widehat{f * g}=\hat{f} \hat{g}$. Furthermore Fourier transformation commutes with complex conjugation. Secondly we have the Gauß functions

$$
f_{S}(a)=i^{S[a]}
$$

for any symmetric matrix $S \in \operatorname{Mat}_{g, g}(\mathbf{Z})$ (here $a$ is regarded as a vector with entries 0,1 and $S[a]={ }^{t} a S a$ is the standard quadratic form). The function $f_{S}$ is a character of second degree in the sense of Weil [W]. The functions $f_{s}^{2}=f_{2 S}$ are characters in the usual sense. We refer for general facts to [W]. Let denote $D_{g}$ the abelian group generated by all Gauß functions and let $D_{g, g}=D_{g} /\left\langle f^{2}\right.$ for $f \in$ $\left.D_{g}\right\rangle$ be the factor group regarded as functions $f_{S}$ with $S$ containing only 0 and 1 . We call these functions $f_{S}$ pure. We include the proof of the following lemma, although elementary, because it is essential for the decomposition.

Lemma 2.1. If $\hat{f}_{S}(a) \in\{0, \pm 1, \pm i\}$ for all $a \in \mathbf{F}_{2}^{g}$ and $\hat{f}_{S}(a)_{a \in \mathbf{F}}$, regarded as vector in $\mathbf{C}^{2^{g}}$, has length 1 , then $S \equiv 0(\bmod 2)$, hence $f_{S}$ is a character on $\mathbf{F}_{2}^{\mathbf{g}}$ with values in $( \pm 1)$.

Proof. We first compute for $f=f_{S}$

$$
\begin{aligned}
f * \bar{f}(a) & =2^{-g} \sum_{b \in \mathbf{F}_{2}^{g}} f(b) \bar{f}(a-b)=2^{-g} \sum_{b \in \mathbf{F}_{2}^{g}} i^{S[b]}(-i)^{S[a-b]} \\
& =(-i)^{S[a]} 2^{-g} \sum_{b \in \mathbf{F}_{2}^{g}}(-1)^{\langle S a, b\rangle} \\
& = \begin{cases}(-i)^{S[a]} & \text { if } S a \equiv 0 \bmod 2 \\
0 & \text { else. }\end{cases}
\end{aligned}
$$


Hence

$$
\begin{aligned}
\bar{f} \overline{\tilde{f}}(a) & =\widehat{f * \bar{f}}(a)=2^{-g} \sum_{b \in \mathbf{F}_{2}^{g}} \sum_{\text {with } S b \equiv 0(2)}(-1)^{\langle b, S b / 2+a\rangle} \\
& \leq 2^{d-g}
\end{aligned}
$$

where $d=\operatorname{dim}(\operatorname{ker}(S))$ regarded as matrix in $\operatorname{Mat}_{g, g}\left(\mathbf{F}_{2}\right)$. The assumption implies that $d=g$ hence the result.

Now we give a matrix description. The Fourier transformation corresponds (up to some scalar factor) to

$$
T_{g}=\left(\frac{1+i}{2}\right)^{g}\left((-1)^{\langle a, b\rangle}\right)_{a b \in \mathbf{F}_{2}^{g}}
$$

and the Gauß functions correspond to

$$
D_{S}=\operatorname{diag}\left(i^{S[a]} \text { for } a \in \mathbf{F}_{2}^{g}\right)
$$

for any symmetric $g \times g$-matrix $S$ with integer coefficients.

Let $H_{g}$ be the subgroup of $G l(V)$ generated by $T_{g}$ and the $D_{S}$, where $S$ runs over the set of symmetric $g \times g$-matrices.

However, for our purpose we need a more combinatorical approach. This leads to a second description of the group $H_{g}$. We regard the affine linear group

$$
A G l\left(g, \mathbf{F}_{2}\right):=\mathbf{F}_{2}^{g} \rtimes G l\left(g, \mathbf{F}_{2}\right)
$$

together with the standard action $(x, M)(a)=M a+x$ on $\mathbf{F}_{2}^{g}$. The group $A G l\left(g, \mathbf{F}_{2}\right)$ will be considered as a subgroup in $G l(V) \cong G l\left(2^{g}, \mathbf{C}\right)$ just by permuting the $f_{a}$.

We recall the following facts $[\mathrm{R}]$ :

We defined $N_{g}=\left\langle D_{S}^{2}, T_{g}^{-1} D_{S}^{2} T_{g}\right.$ for all $\left.S\right\rangle$, the abelian subgroup $D_{g}=\left\langle D_{S}\right.$ for all $S\rangle$ and the subgroup $H_{g, 4}=D_{g} * \operatorname{AGl}\left(g, \mathbf{F}_{2}\right)$. The notation $H_{g, 4}$ is motivated by the fact, that $H_{g, 4}$ is acting on the fourth powers $f_{a}^{4}$ only by permutations. It is a monomial group, regarded as subgroup in $U\left(2^{g}, \mathbf{Z}\left[\frac{1+i}{2}\right]\right) \subset G l\left(2^{g}, \mathbf{C}\right.$ ) (see [R], 2.6). In particular, the entries of a matrix in $H_{g, 4}$ are integral over $\mathbf{Z}$ and of absolute value 1 or 0 , hence contained in $\{0, \pm 1, \pm i\}$. The group $N_{g}$ is a normal subgroup in $H_{g}$ and $N_{g} \subset H_{g, 4}$. One has an exact diagram

$$
\begin{aligned}
& 0 \rightarrow N_{g} \rightarrow H_{g} \rightarrow S p\left(2 g, \mathbf{F}_{2}\right) \rightarrow 0 \\
& \cup \quad U \quad U \\
& 0 \rightarrow \mathbf{F}_{2}^{g} \rightarrow A G l(g) \rightarrow G l\left(g, \mathbf{F}_{2}\right) \rightarrow 0
\end{aligned}
$$


and the index of $H_{g, 4}$ in $H_{g}$ is $1 \cdot 3 \cdot 5 \cdot \ldots \cdot\left(2^{g}+1\right)$.

We have to consider partial Fourier transformation $M$ with respect to the standard flag of subvector spaces in $\mathbf{F}_{2}^{g}$. In matrix notation with respect to the first standard vector (one digit, if one considers $\mathbf{F}_{2}^{g}$ as binary numbers)

$$
M=\frac{1+i}{2}\left(\begin{array}{ccccc}
1 & 1 & & 0 & \\
1 & -1 & & & \\
& & 1 & 1 & \ldots \\
0 & 1 & -1 & \vdots & \ddots
\end{array}\right)
$$

is the matrix with $2^{g-1}$ blocks $\left(\begin{array}{cc}1 & 1 \\ 1 & -1\end{array}\right)$ along the diagonal. The typical Gauß function corresponds to the diagonal matrix (with $2^{g}$ entries)

$$
E=(1, i, 1, i, \ldots) \text {. }
$$

Then $P=i M E^{2} M=(0,1) \ldots\left(2^{g}-2,2^{g}-1\right)$ (in cycle notation) is a permutation in $A G l\left(g, \mathbf{F}_{2}\right)$. We have the relations: $M E M=E M E P,(M E M)^{2}=-P$, $M E^{2} M=i P, M^{2}=i,(M E)^{3}=-1, M E^{2}=P M, E^{2} M=M P, P^{2}=1=E^{4}=$ $(P M)^{4}$. This is easily reduced to a $G l(2, \mathbf{C})$-computation.

More generally we have to regard $d$-dimensional partial Fourier transformation $M^{(d)}$ with respect to the first $d$ standard basis vestors. In matrix notation $M^{(d)}$ for $d \leq g$ is given by the matrix, which consists of $2^{g-d}$ blocks $T_{d}$ along the diagonal (for example in case $d=g=2$

$$
\left.M^{(2)}=\frac{i}{2}\left(\begin{array}{cccc}
1 & 1 & 1 & 1 \\
1 & -1 & 1 & -1 \\
1 & 1 & -1 & -1 \\
1 & -1 & -1 & 1
\end{array}\right)\right)
$$

Let denote by $\Delta_{d, g}: H_{d} \rightarrow H_{g}$ the standard diagonal embedding, which maps a matrix $W$ to the matrix with $2^{g-d}$ blocks $W$ along the diagonal. Then $M^{(d)}=\Delta_{d, g}\left(T_{d}\right)$, $M=\Delta_{1, g}\left(T_{1}\right)$. Let denote by $D_{d, g}$ the set of diagonal matrices in $D_{g}$, given by elements $D_{S}$ with $S$ of the form $\left(\begin{array}{cc}S^{\prime} & 0 \\ 0 & 0\end{array}\right)$ for a symmetric $d \times d$-block $S^{\prime}$ with entries 0 and 1 . The cardinality of this set (of pure Gauß functions belonging to

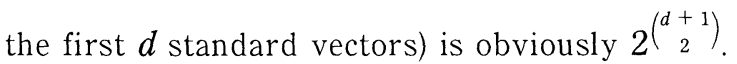

More general, it is possible to define partial Fourier transformation for any linear subspace $U \subset \mathbf{F}_{2}^{g}$. The group $G l\left(g, \mathbf{F}_{2}\right)$ acts by conjugation on the sets $D_{d, g}$ 
and the elements $M^{(d)}$. We get for any $d$-dimensional $U \subset \mathbf{F}_{2}^{g}$ an element $M^{U}$ and a set $D_{g}^{U}$. The elements $M^{U}$ are not unique (depending on a choice of a basis), but nevertheless we get the following result of Bruhat type

THEOREM 2.2. There is a decomposition

$$
H_{g}=\biguplus_{U \subset \mathbf{F}_{2}^{g}} D_{g}^{U} * M^{U} * D_{g} * A I l\left(g, \mathbf{F}_{2}\right) .
$$

Proof. Remember $H_{g, 4}=D_{g} * A G l\left(g, \mathbf{F}_{2}\right)$. Furthermore

$$
\begin{gathered}
\#\left\{d \text {-dim subspaces } \mathrm{U} \text { in } \mathbf{F}_{2}^{g}\right\}=\frac{\left(2^{g}-1\right)\left(2^{g-1}-1\right) \ldots\left(2^{g-d+1}-1\right)}{\left(2^{d}-1\right)\left(2^{d-1}-1\right) \ldots 1}, \\
\#\left\{\text { pure Gauß functions in } D^{U}\right\}=2^{\left(\begin{array}{c}
d+1 \\
2
\end{array}\right)} \text { for } d \geq 1 .
\end{gathered}
$$

Induction shows

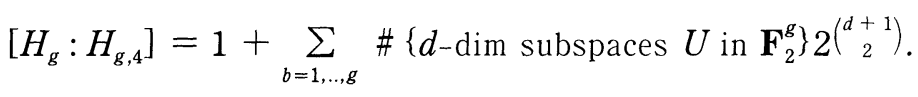

Hence it only remains to show the disjointness of the decomposition. The element $M^{U}$ is clearly unique, it corresponds to a block decomposition of the matrix. Hence we may assume that $M^{U}=M^{(d)}$ (the standard flag). We regard two product representations $D_{1} M^{(d)} B_{1}=D_{2} M^{(d)} B_{2}$ with $D_{i} \in D_{d, g}$ and $B_{i} \in H_{g, 4}$. Obviously is again $D_{1} D_{2}^{-1} \in D_{d, g}$ (up to a character) a pure Gauß function and nontrivial for $D_{1}$ $\neq D_{2}$. Using the maps $\Delta_{d, g}$ it remains to show the following lemma.

Lemma 2.3. It holds $\left[D_{S}, T_{g}\right] \in H_{g, 4}$ iff $S \equiv 0 \bmod 2$.

Proof. It is obvious that $\left[D_{S}^{2}, T_{g}\right] \in H_{g, 4}$. On the other hand we have for the commutator

$$
\left[D_{s}, T_{g}\right]\left(f_{a}\right)=\sum_{c \in \mathbf{F}_{2}^{g}} K_{a, c} f_{c}=\sum_{c \in \mathbf{F}_{2}^{g}}(-1)^{\langle a+c, c\rangle} \overline{\bar{f}}_{s}\left(a+c+S_{c}\right) f_{c} .
$$

If the commutator (i.e. $\left(K_{a, c}\right)$ ) is a monomial matrix, it follows from Lemma 2.1 that $S \equiv \bmod 2$ hence $D_{S} \in N_{g}$.

COROLlaRY 2.4. We have the following equality of groups

$$
H_{g}=\langle G l(g), P, E, M\rangle \text { and } H_{g, 4}=\langle G l(g), P, E\rangle .
$$

$H_{g, 4}$ is the monomial subgroup of $H_{g}$, i.e. the group of all matrices in $\operatorname{Gl}\left(2^{g}, \mathbf{C}\right) \cap H_{g}$ which have exactly one nonzero entry in every row and column. 
The dimension $d$ of the cells in the decomposition are characterized by the property, that the number of nonzero entries in the matrices is just $2^{g-d} 4^{d}$. Dividing by $N_{g}$ gives the Bruhat decomposition with respect to the parabolic subgroup $G l\left(g, \mathbf{F}_{2}\right)$ (corresponding to the set of shorter roots in the Dynkin diagram $C_{g}$ ).

\section{The computation of rings of modular forms}

We recall from $[R]$ the group homomorphism

$$
\phi: \Gamma_{g} \rightarrow H_{g} /( \pm 1) .
$$

For any congruence subgroup $\Gamma$, such that there exists a subgroup $G \subset H_{g}$ with $\Gamma_{g}^{*}(2,4) \subset \Gamma \subset \Gamma_{g}$ and $\phi(\Gamma)=G /( \pm 1)$, we can compute the ring of Siegel mod. ular forms of level $\Gamma$ and even weight as

$$
A(\Gamma)_{(2)}=\bigoplus_{2 \mid s}[\Gamma, s]=\left(\mathbf{C}\left[f_{a} \text { for } a \in \mathbf{F}_{2}^{g}\right]^{G} /\{\text { relations }\}\right)^{N},
$$

where $\mathrm{N}$ denotes the normalization in its field of fractions and "relations" are the theta relations. In genus one and two, there are no relations and in genus three the relations form a principal ideal generated by a polynomial $\phi$ of degree 16 with 471 terms (see Chapter 5). For $g \leq 3$ there is no normalization necessary [loc. cit.]. We get for the invariant rings $R=\mathbf{C}\left[f_{a}\right]^{G}$ the dimension formula:

$$
\Phi_{G}(\lambda)=\sum_{l \geq 0} \operatorname{dim}_{\mathbf{C}} R_{(l)} \times \lambda^{l}=\frac{1}{\# G} \sum_{\sigma \in G} \frac{1}{\operatorname{det}(1-\lambda \sigma)}
$$

(We remark that the degree of a polynomial in the invariant ring is always two times the weight as a modular form.)

Before giving generators for the invariant rings we make some general remarks (see also Stanley [S1], [S2]). All subgroups $G$ of $H_{g, 4}$ are monomial groups (in the matrix representation with the basis $f_{a}$ there is one nonzero entry in every row and column). We have a homomorphism $\pi: G \rightarrow S_{2^{g}}$ (the group of permutation matrices in $G l\left(2^{g}, \mathbf{C}\right)$ ), the group $\operatorname{ker}(\pi)$ is an abelian group consisting of diagonal matrices and $G$ is the semi direct product of $\operatorname{ker}(\pi)$ and $\pi(G)$. All invariants are given as symmetrization (over $\operatorname{im}(\pi)$ in $S_{2^{g}}$ ) of admissible monomials (i.e. invariant under the group $\operatorname{ker}(\pi))$. We fix the notation

$$
\left(b_{0}, \ldots, b_{2^{g}-1}\right)=\frac{1}{\# \operatorname{Stab}\left(b_{0}, \ldots, b_{2^{g}-1}\right)} \sum_{\sigma \in \pi(G)}\left(\prod_{a \in \mathbf{F}_{2}^{g}} f_{a}^{b_{a}}\right)^{\sigma}
$$

where $\left(b_{0}, \ldots, b_{2^{g_{-1}}}\right)$ is $\operatorname{ker}(\pi)$-invariant and the stabilizer is taken inside $G$. It 
will always be clear from the context, if $\left(b_{0}, \ldots, b_{2^{g}}\right)$ denotes a (usually very large) homogeneous polynomial in $\mathbf{C}\left[f_{a}\right]$ or the monomial $\Pi_{a \in \mathbf{F}_{2}^{g}} f_{a}^{b_{a}}$.

The full modular group $\Gamma_{g}$ corresponds to $H_{g}$, which is generated by the monomial group $H_{g, 4}$ and one more element (e.g. $M$ ). In this case invariants will al ways be given as linear combinations of invariants under $H_{g, 4}$.

For groups generated by pseudo reflections the invariant ring is a polynomial ring ([S2], 4.1). In this case it is enough to find $2^{g}$ algebraic independent polynomials in degrees given by the Poincaré-series.

For arbitrary groups it is more difficult to compute the invariant ring. The first step is to find $2^{g}$ algebraic independent invariants in degrees given by the denominator of some representation of the Poincaré-series in the following form.

$$
\Phi_{G}(\lambda)=\frac{1+\sum_{i=2^{g}+1 \ldots . \cdot} \lambda^{d_{i}}}{\Pi_{i=1 \ldots 2^{8}}\left(1-\lambda^{d_{i}}\right)}
$$

This representation is not unique, but if one can find polynomials $g_{1}, \ldots, g_{2^{g}}$ of degree $d_{1}, \ldots, d_{2^{g}}$ with only finitely many common zeros, there also exist $g_{2^{g}+1}, \ldots, g_{r}$ which generate the invariant ring as a free module over the ring $\mathbf{C}\left[g_{1}, \ldots, g_{2^{g}}\right]$ (see $[\mathrm{S} 2]$, Chapter 3 ). The invariant ring is always Cohen-Macaulay (loc. cit. 3.2.).

To find a minimal set of generators as a ring one has to check up to the degree of the numerator that all invariants are polynomials in the chosen set of invariants.

If the invariant ring is a complete intersection

$$
R=\mathbf{C}\left[g_{1}, \ldots, g_{2^{8}+c}\right] /\left(h_{1}, \ldots, h_{c}\right)
$$

(a hypersurface for $c=1$ ), the Poincaré-series has the form

$$
\Phi_{G}(\lambda)=\frac{\Pi_{i=1 \ldots c}\left(1-\lambda^{e_{i}}\right)}{\Pi_{i=1 . .2^{g}+c}\left(1-\lambda^{d_{i}}\right)}
$$

with $g_{i}$ and $h_{\imath}$ polynomials of degree $d_{i}$ and $e_{i}$ respectively ( $c$ is the codimension, see [S1], 3.3). In particular the Poincaré-series is a quotient of products of cyclotomic polynomials.

\section{The Poincaré-series}

The computation of the Poincaré-series is done in two steps. The first step is to compute the Poincaré-series for the action of $H_{3}$ on the polynomial ring, the second is to multiply this series with $\left(1-\lambda^{16}\right)$ or $\left(1-\lambda^{8}\right)$ according to degree or 
weight-grading.

As described above we have to compute many determinants. For genus three there are 371589120. The first simplification we use comes from computing mod ulo the center of the group.

The main simplification comes from the decomposition theorem 2.2. Obviously

$$
\begin{gathered}
\operatorname{det}\left(1-\lambda \sigma_{1} \sigma_{2}\right)=\operatorname{det}\left(1-\lambda \sigma_{2} \sigma_{1}\right) \\
\operatorname{det}(1-\lambda \sigma)=\operatorname{det}\left(\sigma^{-1}-\lambda\right) \operatorname{det}(\sigma) .
\end{gathered}
$$

For $g \geq 3$ the group $H_{g}$ is a subgroup of $S U\left(2^{g}\right)$, moreover $\left(M^{(d)}\right)^{2}=i^{d}$. Let de note $a_{0,3}=1, a_{1,3}=14, a_{2,3}=56, a_{3,3}=64$. All together we get

$$
\begin{aligned}
\Phi_{H_{g}}(\lambda) & =\sum_{l \geq 0} \operatorname{dim}_{\mathrm{C}} R_{(l)} \times \lambda^{l}=\frac{1}{\# H_{g}} \sum_{\sigma \in H_{g}} \frac{1}{\operatorname{det}(1-\lambda \sigma)} \\
& =\frac{1}{\# H_{g}} \sum_{d=0, ., g} a_{d, g} \sum_{\tau \in H_{g, 4}} \frac{1}{\operatorname{det}\left(M^{(d)} \tau-\lambda\right)}
\end{aligned}
$$

It is interesting to note, that we do not need the full knowledge of the conjugacy-classes of $H_{g}$.

This restricts the computation considerably. Only 2752512 determinants of size $8 \times 8$ remain. A tedious computation gives 145 types of characteristic polynomials. In an appendix we give a complete list.

Because $H_{3}$ is a subgroup of $S U(8)$ all the characteristic polynomials P fulfill the equation

$$
\overline{P(X)}=X^{8} * P(1 / X) \text {. }
$$

Moreover by the finiteness of the group $\mathrm{H}_{3}$ all the coefficients of a characteristic polynomial are elements in $\mathbf{Z}[i]$, hence all characteristic polynomials are determined by 7 integers.

For any characteristic polynomial $P$ also $P(i X), P(-X)$ and $P(-i X)$ occur with the same cardinality. The symmetrization over the center

$$
S(P)=1 / P(X)+1 / P(i X)+1 / P(-X)+1 / P(-i X)
$$

yields 33 types of rational function, where the denominator is a product of cyclotomic polynomials $C$, of degree at most 16 and $j$ the order of an element of $H_{g}$ hence a divisor of $371589120=2^{17} * 3^{4} * 5 * 7$.

The set of possible characteristic polynomials is more or less described by this observations. The difficult point is to count how often the 145 types of polynomials occur. 
Before giving the main result for the full modular group let us discuss the easier cases of level 2 and Hecke case. (The Hecke subgroup $\Gamma_{g, 0}(2)$ is for $M=\left(\begin{array}{cc}A & B \\ C & D\end{array}\right) \in \Gamma_{g}$ defined by the condition $C \equiv 0 \bmod (2)$ and corresponds to $H_{g, 4}$ )

The Poincaré-series are as follows:

$$
\begin{aligned}
& \begin{array}{l}
\Phi_{N_{3}}\left(\lambda^{1 / 2}\right) \\
=\frac{1+7 \lambda^{2}+43 \lambda^{4}+154 \lambda^{6}+43 \lambda^{8}+7 \lambda^{10}+\lambda^{12}}{\left(1-\lambda^{2}\right)^{8}} \\
=1+15 \lambda^{2}+135 \lambda^{4}+870 \lambda^{6}+3993 \lambda^{8}+ \\
\quad+14157 \lambda^{10}+41535 \lambda^{12}+105740 \lambda^{14}+\ldots
\end{array}
\end{aligned}
$$

As a consequence one has to compute the invariants up to weight 12 , i.e. with polynomials of degree 24 in the $8 f_{0}, \ldots, f_{7}$. The invariant ring is not a complete intersection (the numerator is irreducible in $\mathbf{Q}[\lambda]$ and not cyclotomic).

Multiplication with $\left(1-\lambda^{8}\right)$ yields:

$$
\begin{aligned}
& \Phi_{\Gamma_{3}(2)}(\lambda) \\
& =\frac{\left(1+7 \lambda^{2}+43 \lambda^{4}+154 \lambda^{6}+43 \lambda^{8}+7 \lambda^{10}+\lambda^{12}\right)\left(1+\lambda^{2}\right)\left(1+\lambda^{4}\right)}{\left(1-\lambda^{2}\right)^{7}} \\
& =1+15 \lambda^{2}+135 \lambda^{4}+870 \lambda^{6}+3992 \lambda^{8}+14142 \lambda^{10}+ \\
& +41400 \lambda^{12}+104870 \lambda^{14}+\ldots
\end{aligned}
$$

For the Hecke subgroup the result is as follows:

$$
\begin{aligned}
\Phi_{H_{3,4}}\left(\lambda^{1 / 2}\right) & =\frac{N_{\text {Hecke }}(\lambda)\left(1+\lambda^{2}\right)}{\left(1-\lambda^{2}\right)\left(1-\lambda^{4}\right)^{3}\left(1-\lambda^{6}\right)\left(1-\lambda^{8}\right)\left(1-\lambda^{12}\right)\left(1-\lambda^{14}\right)} \\
& =1+\lambda^{2}+4 \lambda^{4}+6 \lambda^{6}+15 \lambda^{8}+24 \lambda^{10}+49 \lambda^{12}+78 \lambda^{14}+\ldots
\end{aligned}
$$

The numerator is given by:

$$
\begin{aligned}
& N_{\text {Hecke }}(\lambda)=1-\lambda^{2}+\lambda^{4}+2 \lambda^{8}+\lambda^{10}+2 \lambda^{12}+\lambda^{14}+5 \lambda^{16}+\lambda^{18}+6 \lambda^{20}+2 \lambda^{22} \\
& +6 \lambda^{24}+2 \lambda^{26}+6 \lambda^{28}+\lambda^{30}+5 \lambda^{32}+\lambda^{34}+2 \lambda^{36}+\lambda^{38}+2 \lambda^{40}+\lambda^{44}-\lambda^{46}+\lambda^{48}
\end{aligned}
$$

As a consequence one has to compute the invariants up to weight 50 , i.e. with polynomials of degree 100 in the $8 f_{0}, \ldots, f_{7}$. The invariant ring is not a complete intersection $\left(N_{\text {Hecke }}\right.$ is irreducible in $\mathbf{Q}[\lambda]$ and not cyclotomic).

Multiplication with $\left(1-\lambda^{8}\right)$ yields: 


$$
\begin{aligned}
\Phi_{\Gamma_{3,0}(2)}(\lambda) & =\frac{N_{H \mathrm{ecke}}(\lambda)\left(1+\lambda^{2}\right)}{\left(1-\lambda^{2}\right)\left(1-\lambda^{4}\right)^{3}\left(1-\lambda^{6}\right)\left(1-\lambda^{12}\right)\left(1-\lambda^{14}\right)} \\
& =1+\lambda^{2}+4 \lambda^{4}+6 \lambda^{6}+14 \lambda^{8}+23 \lambda^{10}+45 \lambda^{12}+72 \lambda^{14}+\ldots
\end{aligned}
$$

For the full modular group (or $\mathrm{H}_{3}$ ) the result is as follows:

$$
\begin{aligned}
\Phi_{H_{3}}\left(\lambda^{1 / 2}\right) & =\frac{N_{\text {mod }}(\lambda)\left(1+\lambda^{2}\right)}{\left(1-\lambda^{4}\right)\left(1-\lambda^{8}\right)\left(1-\lambda^{12}\right)^{2}\left(1-\lambda^{14}\right)\left(1-\lambda^{18}\right)\left(1-\lambda^{20}\right)\left(1-\lambda^{30}\right)} \\
& =1+\lambda^{4}+\lambda^{6}+2 \lambda^{8}+2 \lambda^{10}+5 \lambda^{12}+4 \lambda^{14}+\ldots
\end{aligned}
$$

The numerator is given by:

$$
\begin{aligned}
& N_{\text {mod }}(\lambda)=1-\lambda^{2}+\lambda^{4}+\lambda^{10}+3 \lambda^{16}-\lambda^{18}+3 \lambda^{20}+2 \lambda^{22}+2 \lambda^{24}+3 \lambda^{26}+4 \lambda^{28} \\
& +2 \lambda^{30}+7 \lambda^{32}+3 \lambda^{34}+7 \lambda^{36}+5 \lambda^{38}+9 \lambda^{40}+6 \lambda^{42}+10 \lambda^{44}+8 \lambda^{46}+10 \lambda^{48}+ \\
& 9 \lambda^{50}+12 \lambda^{52}+7 \lambda^{54}+14 \lambda^{56}+7 \lambda^{58}+12 \lambda^{60}+9 \lambda^{62}+10 \lambda^{64}+8 \lambda^{66}+10 \lambda^{68}+ \\
& 6 \lambda^{70}+9 \lambda^{72}+5 \lambda^{74}+7 \lambda^{76}+3 \lambda^{78}+7 \lambda^{80}+2 \lambda^{82}+4 \lambda^{84}+3 \lambda^{86}+2 \lambda^{88}+2 \lambda^{90}+ \\
& 3 \lambda^{92}-\lambda^{94}+3 \lambda^{96}+\lambda^{102}+\lambda^{108}-\lambda^{110}+\lambda^{112}
\end{aligned}
$$

To get a numerator with positive coefficients one has to multiply $N_{\text {mod }}(\lambda)$ with $\left(1+\lambda^{2}\right)$. As a consequence one has to compute the invariants at least up to weight 114 , i.e. with polynomials of degree 228 in the $8 f_{0}, \ldots, f_{7}$. The invariant ring is not a complete intersection $\left(N_{\text {mod }}\right.$ is irreducible in $\mathbf{Q}[\lambda]$ and not cyclotomic).

Multiplication with $\left(1-\lambda^{8}\right)$ yields:

$$
\begin{aligned}
\Phi_{\Gamma_{3}}(\lambda) & =\frac{N_{\bmod }(\lambda)\left(1+\lambda^{2}\right)}{\left(1-\lambda^{4}\right)\left(1-\lambda^{12}\right)^{2}\left(1-\lambda^{14}\right)\left(1-\lambda^{18}\right)\left(1-\lambda^{20}\right)\left(1-\lambda^{30}\right)} \\
& =1+\lambda^{4}+\lambda^{6}+\lambda^{8}+2 \lambda^{10}+4 \lambda^{12}+3 \lambda^{14}+\ldots
\end{aligned}
$$

(One may check that $N_{\text {mod }} *\left(1+\lambda_{2}\right)$ is the same numerator given in Tsuyumine's paper [T1]. The statement in [T1], [T3] and [T4], that the ring is not Cohen-Macaulay is not correct.)

Remark. All the Poincaré-series given in this paper have the property

$$
(-\lambda)^{d} \Phi_{G}(1 / \lambda)=\lambda^{\tau} \Phi_{G}(\lambda)
$$

for some $r \in \mathbf{Z}$ which is equivalent to the Gorenstein-property. It turns out that $r$ is the number of pseudo reflections in $G$. For example for $G \subset \operatorname{Sl}(d, \mathbf{C})$ the in variant rings are Gorenstein-rings [S1], 4.4. and 5.5. or [S2], 8.1). We remark, 
that this is not equivalent to the Gorenstein-property of the Satake compactification as a variety.

\section{The case $\Gamma_{3}(2)$}

In the notation with the $f_{a}$ the relation is a polynomial of degree 16 with 471 terms. We have (recall from part I [R])

$$
\begin{aligned}
\psi=2 & (9,1,1,1,1,1,1,1)-(8,0,0,0,2,2,2,2) \\
& +(6,2,2,2,4,0,0,0)-4(5,5,1,1,1,1,1,1) \\
& -(4,4,4,0,4,0,0,0)-2(4,4,0,0,2,2,2,2) \\
& +16(3,3,3,3,1,1,1,1)-72(2,2,2,2,2,2,2,2),
\end{aligned}
$$

where the writing is given with respect to the monomial group $H_{3,4}$. For every monomial one has to take the orbit under the $A G l\left(3, \mathbf{F}_{2}\right)$-action. For $\Gamma_{3}(2)$-which corresponds to the case $N_{3}$-the permutation part is only an abelian group of order 8 , hence one has to collect the 471 terms to 79 orbits in this case to get a writing in accordance with the convention described in chapter 3 . We have to define the notion of an admissible monomial (i.e. invariance under the kernel of the canonical map $N_{3} \rightarrow S_{8}$ ).

Lemma 5.1. A monomial $\left(b_{0}, b_{1}, b_{2}, b_{3}, b_{4}, b_{5}, b_{6}, b_{7}\right)$ is admissible $\Leftrightarrow$

$$
\begin{aligned}
& \sum_{i=0.7} b_{i}=n=2(\text { weight }) \equiv 0 \bmod (4) \\
& b_{0}+b_{1}+b_{2}+b_{3} \equiv 0 \bmod (2) \\
& b_{0}+b_{1}+b_{4}+b_{5} \equiv 0 \bmod (2) \\
& b_{0}+b_{2}+b_{4}+b_{6} \equiv 0 \bmod (2) .
\end{aligned}
$$

Proof. The point is to find a set of conditions, which uniquely defines a monomial modulo the group action. The group of diagonal matrices in this case is generated by $i$ and the following diagonal matrices:

$$
\begin{aligned}
& (-1,-1,-1,-1,1,1,1,1) \\
& (-1,-1,1,1,-1,-1,1,1) \\
& (-1,1,-1,1,-1,1,-1,1)
\end{aligned}
$$

The computation of all modular forms for a certain weight consists in dividing the set of admissible monomials in orbits under the action of the group $\mathbf{F}_{2}^{3}$ in 
the natural decomposition of $\operatorname{AGl}\left(3, \mathbf{F}_{2}\right)$. We will give the invariants by some choice with respect to the permutation orbit under $\operatorname{AGl}\left(3, \mathbf{F}_{2}\right) . \mathbf{F}_{2}^{3}$ is a normal subgroup of $\operatorname{AGl}\left(3, \mathbf{F}_{2}\right)$ and very orbit under $\operatorname{AGl}\left(3, \mathbf{F}_{2}\right)$ decomposes in orbits under $\mathbf{F}_{2}^{3}$. Zeros are omitted if possible.

\section{weight 2 (degree 4).}

In the following tables we choose in every orbit under $\operatorname{AGl}\left(3, \mathbf{F}_{2}\right)$ a representative (first column) and give the cardinality of the orbit under $\operatorname{AGl}\left(3, \mathbf{F}_{2}\right)$ (second column) and the number of linear independent invariants of that type for $\mathbf{F}_{2}^{3}$ (third column).

$$
\begin{array}{lll}
(4) & 8 & 1 \\
(2,2) & 28 & 7 \\
(1,1,1,1) & 14 & 7
\end{array}
$$

\section{weight 4 (degree 8 ).}

$\begin{array}{llllll}(8) & 8 & 1 & (3,1,1,1,2) & 224 & 28 \\ (6,2) & 56 & 7 & (2,2,2,2) & 14 & 7 \\ (5,1,1,1) & 56 & 7 & (1,1,1,1,2,2) & 84 & 21 \\ (4,4) & 28 & 7 & (2,2,2,0,2,0,0,0) & 56 & 7 \\ (4,2,2) & 168 & 21 & (1,1,1,1,4) & 56 & 7 \\ (3,3,1,1) & 84 & 21 & (1,1,1,1,1,1,1,1) & 1 & 1\end{array}$

It follows from the Poincaré-series that the tables aboves give all the 15 and 135 modular forms, which generate the space of modular forms in weight 2 and 4. To get generators for the ring of modular forms, we take all the 15 forms in weight 2 and the last 15 in weight 4 .

THEOREM 5.2. $A\left(\Gamma_{3}(2)\right)$ is minimally generated by the 30 modular forms

$$
\begin{gathered}
(4,0,0,0,0,0,0,0),(2,2,0,0,0,0,0,0),(2,0,2,0,0,0,0,0),(2,0,0,2,0,0,0,0), \\
(2,0,0,0,2,0,0,0),(2,0,0,0,0,2,0,0),(2,0,0,0,0,0,2,0),(2,0,0,0,0,0,0,2), \\
(1,1,1,1,0,0,0,0),(1,1,0,0,1,1,0,0),(1,1,0,0,0,0,1,1),(1,0,0,1,1,0,0,1), \\
(1,0,0,1,0,1,1,0),(1,0,1,0,1,0,1,0),(1,0,1,0,0,1,0,1),(2,2,2,0,2,0,0,0), \\
(2,2,2,0,0,2,0,0),(2,2,2,0,0,0,2,0),(2,2,2,0,0,0,0,2),(2,2,0,0,2,0,2,0), \\
(2,2,0,0,2,0,0,2),(2,0,0,2,0,2,0,2),(1,1,1,1,4,0,0,0),(1,1,4,0,1,1,0,0), \\
(1,1,4,0,0,0,1,1),(1,4,0,1,1,0,0,1),(1,4,0,1,0,1,1,0),(1,4,1,0,1,0,1,0), \\
(1,4,1,0,0,1,0,1),(1,1,1,1,1,1,1,1) .
\end{gathered}
$$


We start with a lemma. Let denote

$$
F_{b}=\sum_{a \in \mathbf{F}_{2}^{g}} f_{a} f_{a+b} \text { for } b \in \mathbf{F}_{2}^{g} .
$$

If one substitutes the $f_{a}$ by its squares one gets the first eight polynomials of the list. The quadrics $F_{b}$ may simultaneously be brought into normal form by a linear change of variable as described in the proof of the following lemma.

Lemma 5.3. The $F_{b}$ have only 0 as a common zero.

Proof. We have

$$
\begin{aligned}
T_{g}\left(F_{b}\right) & =\left(\frac{i}{2}\right)^{g} \sum_{a \in \mathbf{F}_{2}^{g}}\left(\sum_{c \in \mathbf{F}_{2}^{g}}(-1)^{\langle a, c\rangle} f_{c}\right)\left(\sum_{d \in \mathbf{F}_{2}^{g}}(-1)^{\langle a+b, d\rangle} f_{d}\right) \\
& =\left(\frac{i}{2}\right)^{g} \sum_{a, c, d \in \mathbf{F}_{2}^{g}}(-1)^{\langle a, c+d\rangle+\langle b, d\rangle} f_{c} f_{d} \\
& =i^{g} \sum_{c \in \mathbf{F}_{2}^{g}}(-1)^{\langle b, c\rangle} f_{c}^{2} .
\end{aligned}
$$

The matrix $\left((-1)^{\langle b, c\rangle}\right)_{b, c}$ is regular, hence the result.

Proof. (of the theorem) It is enough to show that the polynomials generate the (polynomial) invariant ring. The first eight polynomials have no common zero except $(0, \ldots, 0)$ by Lemma 5.3 . Hence there exists a set of generators containing the first eight polynomials of the list with degrees given by the Poincaré-series, especially by the numerator in the above form.

One can easily see that all basis invariants in degree 8 , different from the last fifteen generators, are linear combinations in the last fifteen and mixed products of first fifteen generators. The last generator $F=\Pi_{a \in \mathbf{F}_{2}} f_{a}$ plays a special role. $F^{3}$ corresponds to the term $\lambda^{12}$ in the numerator and $F^{2} *$ any modular forms of degree 8 (weight 4 ) (and degree 4 (weight 2)) correspond to the term $7 \lambda^{10}$ (and $43 \lambda^{8}$ ) in the numerator of the Poincaré-series. Hence the following problem of linear algebra remains: Is it possible to write all of the 870 basis invariants in degree 12 as a linear combination in the $135 * 15=2025$ products of basis invariants of degree 8 and 4 ? With a computer or with tedious computations one can check that the answer is yes. 


\section{The characterization of decomposable points}

In this chapter we want to derive some consequences for the set of decomposable points from the theory developed in part I, in particular Propositions 2.9, 2.10 , and 3.15 .

As a point set the Satake compactification is given by $\operatorname{Proj}\left(\mathbf{C}\left[f_{a}\right]\right)$. For every $r+s=g$ one has a commutative diagram

$$
\begin{array}{ccccc}
\mathscr{A}_{r}(2,4) & \times \mathscr{A}_{s}(2,4) & \rightarrow & \mathscr{A}_{g}(2,4) \\
& \downarrow & & & \downarrow \\
\mathbf{P}^{2^{r}-1} & \times & \mathbf{P}^{2^{s}-1} & & \rightarrow \mathbf{P}^{2^{g_{-}}}
\end{array}
$$

The map in the upper row is usually denoted by $\Psi$. The map in the lower row is the Segre map. It is well known that the Segre map is an embedding (see [Ha], Ex. I, 2.14).

One may check quite easily that the diagram is equivariant under the various groups $N_{r}, N_{s}$ and $N_{g}$. Hence we get the following

THEOREM 6.1. There is a fibre product diagram of injective maps for every $r+s$ $=g$ as follows

$$
\begin{array}{lllll}
\mathscr{A}_{r}(2) & \times & \mathscr{A}_{s}(2) & \rightarrow & \mathscr{A}_{g}(2) \\
& \downarrow & & & \downarrow \\
\mathbf{P}^{2^{r}-1} / N_{r} & \times & \mathbf{P}^{2^{s}-1} / N_{s} & \rightarrow & \mathbf{P}^{2^{g}-1} / N_{g} .
\end{array}
$$

For $r=s=1$ the Segre embedding is given by the principal ideal $\left(f_{0} f_{3}-\right.$ $f_{1} f_{2}$ ), therefore it is easy to compute the ring of modular forms in genus two by restriction to the diagonal as it is done in Freitag [F](III, 1.4).

For higher genus there are different types of decomposable points given by different types of Segre embeddings. Let $a_{s}$ denote the ideal of a Segre embedding $S$.

COROLlary 6.2 .

$$
\operatorname{Th}\left(\mathscr{A}_{g}(2)^{\text {decomposabie }}\right)=\underset{S \text { Segre map }}{U} Z\left(\mathfrak{a}_{S}^{N_{g}}\right)
$$

where the index set are the various Segre maps. 
Example 6.3. In genus 3 the ideal of one Segre embedding is given by $a_{S}=$ $\left(f_{0} f_{5}-f_{1} f_{4}, f_{2} f_{7}-f_{3} f_{6}, f_{0} f_{6}-f_{2} f_{4}, f_{1} f_{7}-f_{3} f_{5}, f_{0} f_{7}-f_{3} f_{4}, f_{1} f_{6}-f_{2} f_{5}\right)$ and the group $N_{3}$ is acting on the generators by transpositions and multiplication with \pm 1 . By lifting the generators of $A_{2}(2) \times A_{1}(2)$ to $A_{3}(2)$ and computing a basis of $\mathfrak{a}_{\mathrm{S}}^{N_{3}}$ one has another possibility for computing $A_{3}(2)$.

The cartesian product of two graded rings $R_{*}$ and $S_{*}$ is given by $(R \times S)_{i}$ $=R_{i} \otimes S_{i}$ (see [Ha], II. Ex. 5.11) and is a direct summand of the tensor product (with half of the grading). For the groups $N_{r}, N_{s}$ and $N_{g}$ the group action may be extended to the tensor product of the graded rings.

Remark 6.4. In [SM1], [SM2] Salvati Manni gives a characterization of decomposable points in terms of theta constants (of the first kind).

\section{Table}

We give a list of all characteristic polynomials of elements in $H_{3}$ and count their occurrence. The polynomials have the type

$$
P(X)=1+a_{1} X+a_{2} X^{2}+a_{3} X^{3}+a_{4} X^{4}+a_{5} X^{5}+a_{6} X^{6}+a_{7} X^{7}+X^{8}
$$

with $\overline{a_{1}}=a_{7}, \overline{a_{2}}=a_{6}, \overline{a_{3}}=a_{5}$ and $a_{4} \in \mathbf{Z}$. This table is included to allow comparison with other approaches.

In the table, after the coefficients, we give the number of types under the action of the center (a row may belong to at most four characteristic polynomials) and the numbers corresponding to the $d$-dimensional $M^{(d)}$, the number of cases is the sum \#\{0-dim cases $\}+14 * \#\{1$-dim cases $\}+56 * \#\{2$-dim cases $\}+64 *$ $\#\{3$-dim cases $\}$. The last column gives the product decomposition into cyclotomic polynomials of the denominator of the symmetrization $\mathrm{S}(\mathrm{P})$. There are different symmetrizations with equal cyclotomic type. By summation over the 0-dimensional cases one gets the Poincaré-series for $H_{3,4}$.

$\begin{array}{llllllllll}a_{1} & a_{2} & a_{3} & a_{4} & \# & 0-\operatorname{dim} & \text { 1-dim } & \text { 2-dim } & \text { 3-dim } & \text { cyclotomic type } \\ i & 0 & 0 & 0 & 4 & 196608 & 49152 & 122880 & 86016 & 28,14,7,4^{2}, 2^{2}, 1^{2} \\ 0 & 0 & 0 & 2 & 1 & 193536 & 62976 & 44544 & 34944 & 8^{2} \\ 0 & 2 i & 0 & -2 & 2 & 16128 & 13824 & 4608 & 4032 & 8^{2} \\ 1+i & 0 & 1-i & 2 & 4 & 21504 & 39936 & 16896 & 21504 & 8^{2} \\ 0 & 0 & 0 & -2 & 1 & 246456 & 34848 & 56640 & 29568 & 4^{2}, 2^{2}, 1^{2} \\ 0 & 2 & 0 & 2 & 2 & 32256 & 9216 & 13824 & 8064 & 4^{2}, 2^{2}, 1^{2} \\ 1+i & 2 i & -1+i & -2 & 4 & 21504 & 3072 & 26112 & 21504 & 4^{2}, 2^{2}, 1^{2} \\ 2 & 2 & 2 & 2 & 4 & 16128 & 4608 & 6912 & 4032 & 4^{2}, 2^{2}, 1^{2} \\ 2 & 4 & 6 & 6 & 4 & 1344 & 384 & 192 & 672 & 4^{2}, 2^{2}, 1^{2}\end{array}$




\begin{tabular}{|c|c|c|c|c|c|c|c|c|c|}
\hline $2-2 i$ & $-4 i$ & $-2-2 i$ & -2 & 4 & 1568 & 512 & 128 & 224 & $4^{2}, 2^{2}, 1^{2}$ \\
\hline 0 & 1 & 0 & 0 & 2 & 172032 & 36864 & 55296 & 21504 & $12,6,4^{2}, 3,2^{2}, 1^{2}$ \\
\hline 0 & $i$ & 0 & 0 & 2 & 114688 & 90112 & 61440 & 86016 & $24,8^{2}$ \\
\hline 0 & 0 & 0 & 0 & 1 & 172032 & 172032 & 172032 & 172032 & 16 \\
\hline $1+i$ & $i$ & $1-i$ & 2 & 4 & 28672 & 4096 & 11264 & 8960 & $12,6,4^{2}, 3,2^{2}, 1^{2}$ \\
\hline $1+i$ & $i$ & $-1+i$ & -2 & 4 & 28672 & 40960 & 19456 & 34048 & $12,6,3$ \\
\hline 0 & 0 & 0 & 1 & 1 & 0 & 179712 & 40704 & 91392 & $12,6,3$ \\
\hline 1 & 1 & 0 & 0 & 4 & 0 & 61440 & 36864 & 75264 & $12,6,3$ \\
\hline $1+i$ & $2 i$ & $-2+2 i$ & -3 & 4 & 0 & 9216 & 13824 & 16128 & $12,6,3$ \\
\hline 0 & $4 i$ & 0 & -6 & 2 & 3584 & 1280 & 256 & 448 & $8^{4}$ \\
\hline 0 & 4 & 0 & 6 & 2 & 5670 & 384 & 480 & 0 & $4^{4}, 2^{4}, 1^{4}$ \\
\hline 2 & 0 & -2 & -2 & 4 & 14784 & 384 & 2112 & 672 & $4^{4}, 2^{4}, 1^{4}$ \\
\hline 2 & 1 & 2 & 4 & 4 & 14336 & 0 & 2560 & 896 & $12^{2}, 6^{2}, 4^{4}, 3^{2}, 2^{4}, 1^{4}$ \\
\hline 2 & 1 & -2 & -4 & 4 & 14336 & 6144 & 6656 & 2688 & $12^{2}, 6^{2}, 4^{2}, 3^{2}, 2^{2}, 1^{2}$ \\
\hline $2+2 i$ & $2 i$ & $2-2 i$ & 6 & 4 & 1344 & 1920 & 192 & 336 & $8^{2}, 4^{2}, 2^{2}, 1^{2}$ \\
\hline $2+2 i$ & $6 i$ & $-6+6 i$ & -10 & 4 & 1344 & 384 & 576 & 336 & $8^{2}, 4^{2}, 2^{2}, 1^{2}$ \\
\hline 4 & 8 & 12 & 14 & 4 & 420 & 48 & 48 & 0 & $4^{4}, 2^{4}, 1^{4}$ \\
\hline 4 & 4 & -4 & -10 & 4 & 364 & 0 & 16 & 0 & $4^{6}, 2^{6}, 1^{6}$ \\
\hline $4+4 i$ & $16 i$ & $-20+20 i$ & -34 & 4 & 14 & 8 & 0 & 0 & $4^{4}, 2^{4}, 1^{4}$ \\
\hline 8 & 28 & 56 & 70 & 4 & 1 & 0 & 0 & 0 & $4^{8}, 2^{8}, 1^{8}$ \\
\hline 0 & 2 & 0 & 3 & 2 & 0 & 12672 & 9408 & 5376 & $12^{2}, 6^{2}, 3^{2}$ \\
\hline 0 & 0 & 0 & -1 & 1 & 0 & 79872 & 89088 & 86016 & 24 \\
\hline 0 & $i$ & 0 & -1 & 2 & 0 & 73728 & 73728 & 64512 & 40 \\
\hline $1+i$ & $i$ & 0 & 1 & 4 & 0 & 36864 & 36864 & 32256 & $20,10,5$ \\
\hline 0 & 1 & 0 & 1 & 2 & 0 & 36864 & 36864 & 32256 & $20,10,5$ \\
\hline 1 & 0 & -1 & -1 & 4 & 0 & 49152 & 49152 & 43008 & $60,30,15$ \\
\hline 0 & $2 i$ & 0 & -3 & 2 & 0 & 15360 & 3584 & 3584 & $24^{2}$ \\
\hline $1+i$ & 0 & 0 & -1 & 4 & 0 & 33792 & 7680 & 16128 & $24,12,6,3$ \\
\hline 2 & 2 & 0 & -1 & 4 & 0 & 5376 & 4224 & 2688 & $12^{2}, 6^{2}, 3^{2}$ \\
\hline 1 & 1 & 1 & 1 & 4 & 0 & 49152 & 73728 & 86016 & $36,18,12,9,6,3$ \\
\hline 1 & 1 & -2 & -2 & 4 & 0 & 2048 & 0 & 1792 & $12^{3}, 6^{3}, 4^{2}, 3^{3}, 2^{2}, 1^{2}$ \\
\hline 2 & 3 & 4 & 5 & 4 & 0 & 6144 & 6144 & 5376 & $20^{2}, 10^{2}, 5^{2}$ \\
\hline 1 & 1 & 2 & 2 & 4 & 0 & 2048 & 12288 & 8960 & $12^{2}, 6^{2}, 4^{2}, 3^{2}, 2^{2}, 1^{2}$ \\
\hline 2 & 4 & 4 & 5 & 4 & 0 & 1792 & 384 & 1792 & $12^{3}, 6^{3}, 3^{3}$ \\
\hline $2+2 i$ & $4 i$ & $-4+4 i$ & -7 & 4 & 0 & 1920 & 448 & 448 & $12^{2}, 6^{2}, 3^{2}$ \\
\hline 4 & 10 & 16 & 19 & 4 & 0 & 64 & 32 & 0 & $12^{4}, 6^{4}, 3^{4}$ \\
\hline
\end{tabular}




\section{Appendix}

As an appendix we give the Poincaré-series and the graded rings of modular forms for genus one and two. In this cases our method reduces to a very simple one. A second method (in level 2) would be just to apply the (surjective) $\Phi$-operator from genus three to get the results in genus one and two. In the following tables we give the congruence subgroup, the order of the corresponding finite subgroup in $H_{g}$, the Poincaré-series and the ring of modular forms for that congruence subgroup. Although the results are known, it may be useful for the applications in coding theory, to give the invariant rings in terms of theta constants of second order (and in genus two the exceptional $\Theta=\Pi_{\text {even } m} \theta[m]$ ).

$$
\begin{array}{llll}
\Gamma_{1}(4) & 2 \frac{1+\lambda}{(1-\lambda)^{2}} & \mathbf{C}[(2,0),(0,2),(1,1)] \\
\Gamma_{1}(2) & 16 \frac{1}{\left(1-\lambda^{2}\right)^{2}} & \mathbf{C}[(4,0),(2,2)] \\
\Gamma_{1,0}(2) & 32 \frac{1}{\left(1-\lambda^{2}\right)\left(1-\lambda^{4}\right)} & \mathbf{C}[(4,0),(4,4)] \\
\Gamma_{1} & 96 \frac{1}{\left(1-\lambda^{4}\right)\left(1-\lambda^{6}\right)} & \mathbf{C}[(8,0)+14(4,4),(12,0)-33(8,4)]
\end{array}
$$

That corresponds to the fact that the three group $N_{1}, N_{1,4}$ and $H_{1}$ are generated by pseudo reflections, whereas $\Gamma_{1}(4)$ corresponds to \pm 1 .

In the genus two case we will always distinguish between the case of even weight and the general case. In the case of even weight we get:

$$
\begin{aligned}
& \Gamma_{2}(2) \quad 32 \quad \frac{1-\lambda^{8}}{\left(1-\lambda^{2}\right)^{5}} \quad \mathbf{C}[(4,0,0,0),(1,1,1,1),(2,2,0,0),(2,0,2,0),(2,0,0,2)] \\
& \Gamma_{2,0}(2) 3072 \frac{\left(1-\lambda^{2}\right)^{5}}{\left(1-\lambda^{2}\right)\left(1-\lambda^{4}\right)^{2}\left(1-\lambda^{6}\right)} \\
& \text { C }[(4,0,0,0),(4,4,0,0),(2,2,2,2),(12,0,0,0)]
\end{aligned}
$$

In the case of general weight we get (following [R], 4.2):

$$
\begin{array}{cl}
\Gamma_{2}(2) \quad 32 \quad \frac{\left(1-\lambda^{8}\right)\left(1+\lambda^{5}\right)}{\left(1-\lambda^{2}\right)^{5}} \\
\Gamma_{2,0}(2) \quad 3072 \quad \frac{\left(1+\lambda^{19}\right)}{\left(1-\lambda^{2}\right)\left(1-\lambda^{4}\right)^{2}\left(1-\lambda^{6}\right)} \\
& \mathbf{C}\left[(4,0,0,0),(4,4,0,0),(2,2,2,2),(12,0,0,0), \Theta * P_{28}\right]
\end{array}
$$


If one denotes

$$
A=(4,0,0,0), B=(1,1,1,1), C=(2,2,0,0), D=(2,0,2,0), E=(2,0,0,2),
$$

then there is a relation of degree 16 between the generators in level 2 as follows.

$$
16 B^{4}+A^{2} B^{2}+C^{2} D^{2}+D^{2} E^{2}+C^{2} E^{2}=A C D E+4 B^{2}\left(C^{2}+D^{2}+E^{2}\right)
$$

Hence $\mathscr{A}_{2}(2)$ is a quartic hypersurface in $\mathbf{P}^{4}$ (see also Igusa [11], [12]).

In level $\Gamma_{2,0}(2)$ the computation is easy because the group $H_{2,4}$ is a group generated by pseudo reflections of order 3072 . Hence $\mathscr{A}_{2,0}(2)$ is a weighted $\mathbf{P}^{3}$ and hence rational (see also Ibukiyama $[\mathrm{Ib} 1,2]$ ). The polynomial $P_{28}$ is given as follows:

$$
P_{28}=f_{0} f_{1} f_{2} f_{3} *\left(f_{0}^{4}-f_{1}^{4}\right)\left(f_{0}^{4}-f_{2}^{4}\right)\left(f_{0}^{4}-f_{3}^{4}\right)\left(f_{1}^{4}-f_{2}^{4}\right)\left(f_{1}^{4}-f_{3}^{4}\right)\left(f_{2}^{4}-f_{3}^{4}\right)
$$

\section{REFERENCES}

[F] Freitag, E., Siegelsche Modulfunktionen, (Grundlehren der math. Wissenschaften, vol. 254), Springer, Berlin Heidelberg-New York, 1983.

[Ha] Hartshorne, R., Algebraic Geometry, Graduate Texts in Math., 52, Springer, New York-Berlin 1977.

[Ib1] Ibukiyama, T., On symplectic Euler factors of genus two, J. Fac. Sci. Univ. Tokyo, Sect. IA Math., 30 (1984), 587-614.

[Ib2] - On Siegel modular varieties of level 3, Internat. J. Math., 2, No. 2 (1991), $17-35$.

[I1] Igusa, J., On Siegel modular forms of genus two, Amer. J. Math., 84 (1962), $175-200$.

[12] - On Siegel modular forms of genus two (2), Amer J. Math., 86 (1964), $392-412$.

[I3] - On the graded ring of theta-constants, Amer J. Math., 86 (1964), $219-246$.

[I4] - On the graded ring of theta constants II, Amer J. Math. 88 (1966), $221-236$.

[R] Runge, B., On Siegel modular forms, part I, J. Reine angew. Math., 436 (1993), $57-85$.

[SM1] Salvati Manni, R., On the projective varieties associated with some subrings of the ring of Thetanullwerte, preprint 1992.

[SM2] - Maps defined by Thetanullwerte, preprint 1992.

[Sh] Shioda, T., On the graded ring of invariants of binary octavics, Amer, J. Math., 89 (1967), $1022-1046$.

[S1] Stanley, R. P., Hilbert functions of graded algebras, Adv. in Math., 28 (1978), $57-83$

[S2] - Invariants of finite groups and their applications to combinatorics, Bull Amer. Math. Soc., 1, 3 (1979), 475-511.

[T1] Tsuyumine, S., On Siegel modular forms of degree three, Amer. J. Math., 108 
(1986), 755-862.

[T2] - Addendum to "On Siegel domular forms of degree three", Amer. J. Math., 108 (1986), $1001-1003$.

[T3] - Rings of automorphic forms which are not Cohen-Macaulay II, J. Math. Soc. Japan, 40 (1988), 369-381.

[T4] - On the Graded Rings of Modular Forms in Several Variables, Adv. Stud. in Pure Math., 15, Automorphic Forms and Geometry of Arithmetic Varieties, (1989), 65-87.

[W] Weil, A., Sur certain groupes d'opérateurs unitaires, Acta Math., 111 (1964), $143-211$

Max-Planck-Arbeitsgruppe

"Algebraische Geometrie und Zahlentheorie"

Jägerstraße 10/11

10117 Berlin

Germany

e-mail: runge@zahlen.ag-berlin.wpg.de 